\title{
Emprendimiento femenino: características, motivos de éxito, limitantes, involucrados y consecuencias
}

\section{Female entrepreneurship: characteristics, reasons for success, limitations, involved and consequences}

Natividad Carmen Orihuela-Ríos

Universidad César Vallejo, Lima, Perú

adrianos2005@hotmail.com

(iD) https://orcid.org/0000-0002-8596-4903

Recepción: 30/09/2021 | Aceptación: 20/12/2021 | Publicación: 10/01/2022

Cómo citar (APA, séptima edición):

Orihuela-Ríos, N.C. (2022). Emprendimiento femenino: características, motivos de éxito, limitantes, involucrados y consecuencias. INNOVA Research Journal, 7(1), 109-122.

https://doi.org/10.33890/innova.v7.n1.2022.1946

\section{Resumen}

El Global Entrepreneurship Monitor Perú 2017 - 2018 explicó que Perú ocupa el primer lugar en América Latina y el Caribe y el quinto en el Índice de Emprendimiento. La presencia de mujeres emprendedoras por oportunidad es de 0,92 por cada hombre, por lo que se incorporaron a la economía del país y están generando empleo directa e indirectamente. El objetivo del estudio fue analizar el emprendimiento femenino e identificar las características, motivos de éxito, limitaciones, implicaciones y consecuencias. El estudio se desarrolló bajo el paradigma interpretativo, enfoque cualitativo, método analítico, sintético e inductivo. La categoría de estudio se dividió en cinco subcategorías, y se codificó cada indicador, luego se trianguló la información de la entrevista con el apoyo del software Atlas.ti 8. La principal característica de las mujeres emprendedoras es la perseverancia y siempre están atentas a las oportunidades del mercado. Una limitación es el acceso al financiamiento para el capital inicial, así como los trámites, son burocráticos. A pesar del carácter exigente del negocio, las mujeres no descuidan sus tareas como madres. La familia apoya el crecimiento y desarrollo de la actividad económica, lo que se traduce en rentabilidad económica y riqueza personal, lo que permite la emprendedora independencia económica y estatus social.

Palabras claves: emprendimiento; mujer; oportunidades de empleo.

Abstract 
The Global Entrepreneurship Monitor Peru 2017 - 2018 explained that Peru ranks 1st in Latin America and the Caribbean, and 5th in the Entrepreneurship Index. The presence of women entrepreneurs by opportunity is 0.92 for each man, which is why they joined the economy of the country and are generating jobs directly and indirectly. The objective of the study was to analyse female entrepreneurship and identify the characteristics, reasons for success, limitations, involved, and consequences. The study was developed under the interpretive paradigm, qualitative approach, analytical, synthetic, and inductive method. The study category was broken down into five subcategories, and each indicator was coded, then the interview information was triangulated with the support of Atlas.ti 8 software. The main characteristic of women entrepreneurs is perseverance and they are always attentive to opportunities from the market. A limitation is an access to financing for the initial capital, as well as the procedures, are bureaucratic. Despite the demanding nature of the business, women do not neglect their tasks as mothers. The family supports the growth and development of the economic activity, which translates into economic profitability and personal wealth, which allows the entrepreneurial economic independence and social status.

Key words: entrepreneurship; woman; job opportunities.

\section{Introducción}

El emprendimiento femenino a través del tiempo se acrecentó, básicamente por la independencia económica que ha perseguido por mucho tiempo la mujer. En Chile se expuso que más del 50\% de las mujeres emprenden por una oportunidad en el mercado, la motivación se circunscribe a la generación de ingresos, apoyo a la familia y facilidad para organizar su tiempo. En el estudio se evidenció que el mayor número de emprendimientos se registran en Arica, Parinacota y Tarapacá, fundamentalmente por la cercanía al puerto y las operaciones de comercio al menudeo (Pontificia Universidad Católica de Chile, 2018). En España menos del 50\% de las mujeres emprende, porque existe el temor al fracaso, lo cual las limita a realizar una nueva actividad (Instituto de Empresa, 2019).

Las mujeres que emprenden lamentablemente tienen que enfrentarse a un sin número de limitantes en el mercado, básicamente porque la sociedad ha otorgado un lugar privilegiado al género masculino por mucho tiempo. Las entidades bancarias y financieras otorgan financiamiento con cautela, y los proveedores desconfían realizar operaciones al crédito (Ascher, 2012). En Saltillo y Torreón las emprendedoras tienen estudios a nivel universitario, se dedican básicamente a la actividad comercial y de servicios, lo gratificante es que lograron socialmente ocupar un lugar, al mismo tiempo han incrementado sus ingresos, se sienten satisfechas, lograron un equilibrio entre la vida de familia y el negocio o empresa (Guerrero, Gómez y Armenteros, 2014).

La investigación tiene como objetivo analizar el emprendimiento femenino e identificar las características, motivos de éxito, limitaciones, implicaciones y consecuencias en Lima, Perú. Según el informe de Global Entrepreneurship Monitor (2017) el emprendimiento femenino de la mujer peruana se realiza en la actividad de servicios, la mayoría posee secundaria incompleta, la generación de empleo directo se ubica entre 5 y 19 colaboradores, sin embargo, una vez más se resalta que el motivo fundamental es la independencia económica. 
Esta situación es muy parecida a Bangladesh, porque dicho grupo humano trabaja con redes para comercializar sus productos (Amin, 2018), en Tanzania el factor influyente es lograr el bienestar personal (Bouzekraoui y Ferhane, 2017). En marroquí se exhibió el hecho que las emprendedoras en su mayoría poseen estudios universitarios, la familia apoya y tiene incidencia directa en las decisiones del negocio, no obstante los problemas que se presentan en Marroquí, están vinculados al financiamiento, la adquisición de maquinaria y equipo, pero sobre todo el acceso al mercado potencial (Bouzekraoui y Ferhane, 2017). Mientras que India el gobierno no implementa estrategias para promover el emprendimiento femenino, a pesar de que son conscientes en el aporte al crecimiento y el desarrollo económico (Kumar, 2015).

\section{Marco teórico}

En la revisión de investigaciones previas, Eib y Sieget (2019) aseveraron que las mujeres en Europa han optado por el empoderamiento económico, sin tener que descuidar sus obligaciones propias del género y responsabilidades, los resultados evidenciaron que los hombres tienen mayor éxito comercial respecto a las mujeres. En este sentido se resalta el hecho que la mujer que emprende nunca descuida las tareas como madre o esposa, por lo tanto, el núcleo familiar persiste y en ocasiones se fortaleza por el aporte económico que realiza a la familia.

Chowdhury (2018) expuso que un problema que enfrentan las mujeres de Bangladesh es el financiamiento, porque existe una brecha con el género masculino, sin embargo las ONGs no las abandonaron, por el contrario, son las que brindan créditos a tasa menor al 10\%, por otra parte, hace falta mentoras y personas responsables en la toma de decisiones, por ello se requiere el apoyo del Estado, más aún desde el Ministerio de Industria y Comercio se debe incentivar no solo el emprendimiento, sino también la formalización de los negocios. Por lo tanto, esta situación conlleva a una oportunidad para las entidades financieras para diseñar productos para el apalancamiento dirigido a las mujeres emprendedoras, de tal forma que el costo que asuman les permita generar rentabilidad y hacer sostenible el emprendimiento.

Jia, Parvin y Rahman (2012) encontraron que los factores con mayor relación con el emprendimiento de mujeres son: la libertad para trabajar, el deseo de mayores ingresos, alcanzar un mayor estatus social, mayores niveles educativos, tamaño de la familia, relaciones familiares, muerte del esposo o cabeza de familia, la motivación de familiares y/o amigos, acceso al crédito y entrenamiento en emprendimiento, apoyo gubernamental, pertenencia a asociaciones de emprendedores, acceso a información del negocio e infraestructura favorable al emprendimiento (Kargwell, 2012). En consecuencia, es importante la inclusión de la mujer en la generación de los recursos económicos, lo que implica los contratos laborales y su situación en la estructura orgánica (Huilcapi, Castro y Jácome, 2017).

En este sentido, el emprendimiento femenino se incrementó a través del tiempo, motivado básicamente por la necesidad de aportar a la economía familiar, sin embargo, el principal impedimento siempre ha sido el capital. Por lo tanto, las entidades financieras han brindado el apalancamiento, el mismo que no ha sido suficiente para el gran número de mujeres, que en muchos de los casos las obligó a recurrir a los prestamistas, asumiendo costos elevados, lo cual afectó a la sostenibilidad del negocio. Por tal motivo, es necesario que las entidades del estado en 
sinergia con la banca privada diseñen productos para las emprendedoras, además del acompañamiento y asesoría para que los negocios crezcan en el tiempo.

Las teorías que sustentan el estudio corresponden a lo expuesto por Mc Clelland con la teoría de rasgos, porque las mujeres tienen como objetivo la independencia económica, el apoyo a su familia y más aún ser reconocidas por la sociedad (Huilcapi, Castro y Jácome, 2017), al mismo tiempo la teoría del cambio institucional de North ayuda a comprender la nueva estructura económica del país, porque las mujeres con las actividades que realizan también aportan al crecimiento económico (Villagómez, 1994).

La educación y capacitación juegan un papel importante, porque permite adquirir nuevas competencias que respondan a las necesidades de la empresa para diseñar planes de mejora, tal como se expresa en la teoría del capital humano (Villalobos y Pedroza, 2009). En este mismo sentido la sinergia entre la innovación y el emprendimiento permite aportar a la sociedad con nuevos productos o servicios, dinamizando la economía en la generación de recursos económicos (Sánchez, 2006), no solo para la familia, sino que a la vez genera puestos de trabajo directos e indirectos.

En tal sentido, las propuestas teóricas han permitido el desarrollo del emprendimiento acorde a los cambios tecnológicos digitales, Gutiérrez (2006) y Drucker (1985) quien este último agrega el término innovador a las características emprendedoras, diferenciándola en gran medida a la persona que mejora de manera sustantiva la organización empresarial, y no solo aquel que apertura un negocio determinado, sino que lo potencializa, lo vuelve óptimo y operativo, predispuesto al cambio y aumento del rendimiento laboral.

El término emprendimiento posee una cantidad sustantiva de definiciones polisémicas, las cuales, coinciden o se diferencian según los puntos de vista. La palabra proviene del francés entrepreneur que hace mención según Nueno (1999) a la "capacidad de una persona para hacer un esfuerzo adicional por alcanzar una meta u objetivo" (p. 23). También el emprendimiento se refiere a la capacidad de visualizar una oportunidad y decidir emprender (McKenzie, Ugbah y Smothers, 2015), para ello se diseñan planes con simulaciones económicas que convenzan al emprendimiento (Da silva, Esper, Bencciveni y Chagas, 2015; Rosa, Souza y Loch, 2011). En tal sentido, en la investigación se asume al emprendimiento como la decisión de la mujer para emprender una actividad económica que le permita generar recursos económicos, los cuales se capitalicen a través del tiempo y permita satisfacer sus necesidades personales, así como las familiares.

Al referirse al emprendimiento femenino, Casson (2001) direcciona a la participación efectiva de las mujeres en las actividades comerciales de innovación comercial. Por ello, son consideradas personas calificadas que crean y gestionan una empresa con el propósito de obtener beneficios, desarrollo y crecimiento, caracterizado por un comportamiento innovador e inclusivo además emplean prácticas de gestión estratégica en la organización empresarial.

Dornela (2001) amplía las características y sostuvo que son optimistas, apasionadas, perseverantes, comprometidas, asumen riesgos, resueltas, dinámicas, fijan sus metas y objetivos, buscan información, independientes, autónomas, tienen autocontrol y crean valor; no obstante Jia, Parvin, y Rahman (2012) identificaron que los factores que están vinculados al emprendimiento 
corresponde a la independencia por el trabajo, generación de ingresos, status social, superarse académicamente, la disolución familiar, el tamaño de la familia y las carencias, empuje del entorno, condiciones para el emprendimiento (Jia, Parvin y Rahman, 2012).

Sin embargo, desde la perspectiva de Vásquez (2016) el emprendimiento se caracteriza por la innovación y adaptación del producto o servicio a las necesidades del mercado, por ende no se concentra solo en gestionar la organización, sino que debe superarla, con el fin de ser sostenible, lo que se resume en un comportamiento diferente a las personas de su entorno (Castillo, 1999), rescatando que el financiamiento es una de las debilidades, sin embargo se rescata el nivel de riesgo que asumen, pero que ambas no sin limitantes, por el contrario ello las motiva a iniciar y seguir avanzando.

Finalmente, dentro de las características de las mujeres emprendedoras se rescata el hecho que son perseverantes, priorizan sus objetivos a pesar del cansancio y el fracaso. Todo ello conlleva a asumir riesgos financieros básicamente y de mercado, el primero por el costo que tiene que asumir por no disponer del capital, y el segundo porque el producto puede no tener aceptación en el mercado o requiere adaptarse a las nuevas necesidades.

\section{Metodología}

El estudio se desarrolló bajo el paradigma interpretativo, se recopiló data que corresponda al fenómeno de estudio del emprendimiento femenino y posee fundamento teórico (Numa y Márquez, 2019; Barco y Carrasco, 2018); es preciso señalar que es un requisito fundamental que el investigador se despoje de sus propios paradigmas, para tener una visión concienzuda del problema.

El enfoque fue cualitativo, los datos se recopilaron a través de las entrevistas semiestructuradas y las respectivas guías de entrevista a las mujeres emprendedoras (Hernández, Fernández y Baptista, 2014). El criterio de inclusión fue: a) Género femenino; b) Haber emprendido un negocio en últimos 5 años; c) El emprendimiento debe estar situado en Villa El Salvador, específicamente en la zona industrial, ubicado al Sur de Lima. En este sentido, es preciso hacer hincapié en el hecho que fue importante lograr la aceptación de las mujeres para participar de manera voluntaria en el estudio, con el fin de lograr la información fidedigna y no se sientan presionadas por compartir su experiencia en el emprendimiento.

En tal sentido, a partir de las entrevistas se comprendió e interpretó cada afirmación según la codificación axial de los indicadores, subcategorías y la categoría de estudio, que para el estudio corresponde al emprendimiento femenino (Mehrad y Tahriri, 2019; Navarro, Jiménez, Rappoport y Thailiez, 2017).

Es así como, una vez obtenida la información tuvo que triangularse, es una técnica de identificación de las coincidencias y diferencias en las afirmaciones según la codificación axial previamente diseñada, luego la organización de estas se realizó en función a los objetivos del estudio, para concatenar y vincular lo vertido por las emprendedoras con las teorías y conceptos de emprendimiento, para informar los hallazgos válidos y consistentes (Okuda y Gómez-Restrepo, 2005). 
También se aplicó diferentes métodos como:

a) Analítico-sintético, de forma simultánea se realiza ambos procesos, partiendo por la descomposición de cada afirmación o frase según los códigos, luego se relacionaron todas las afirmaciones según los códigos apriorísticos como son: SC1 Características de las mujeres emprendedoras; SC2 Motivos de éxito del negocio; SC3 Limitantes para emprender; SC4 Involucrados en el emprendimiento; SC5 Consecuencias del emprendimiento (Ver figura 1), para continuar con el proceso de sinergia y complementariedad de los mismos, para luego integrarlos y responder al objetivo de la investigación que es analizar el emprendimiento femenino (Rodríguez y Pérez, 2017);

b) Inductivo, porque a partir de los resultados que se hallaron en el estudio se infiere que las mujeres emprendieron por necesidad y superación, pero sobre todo para aportar a la economía familiar (Prince y Felder, 2006).

\section{Figura 1}

Categoría, subcategorías e indicadores para medir el emprendimiento femenino

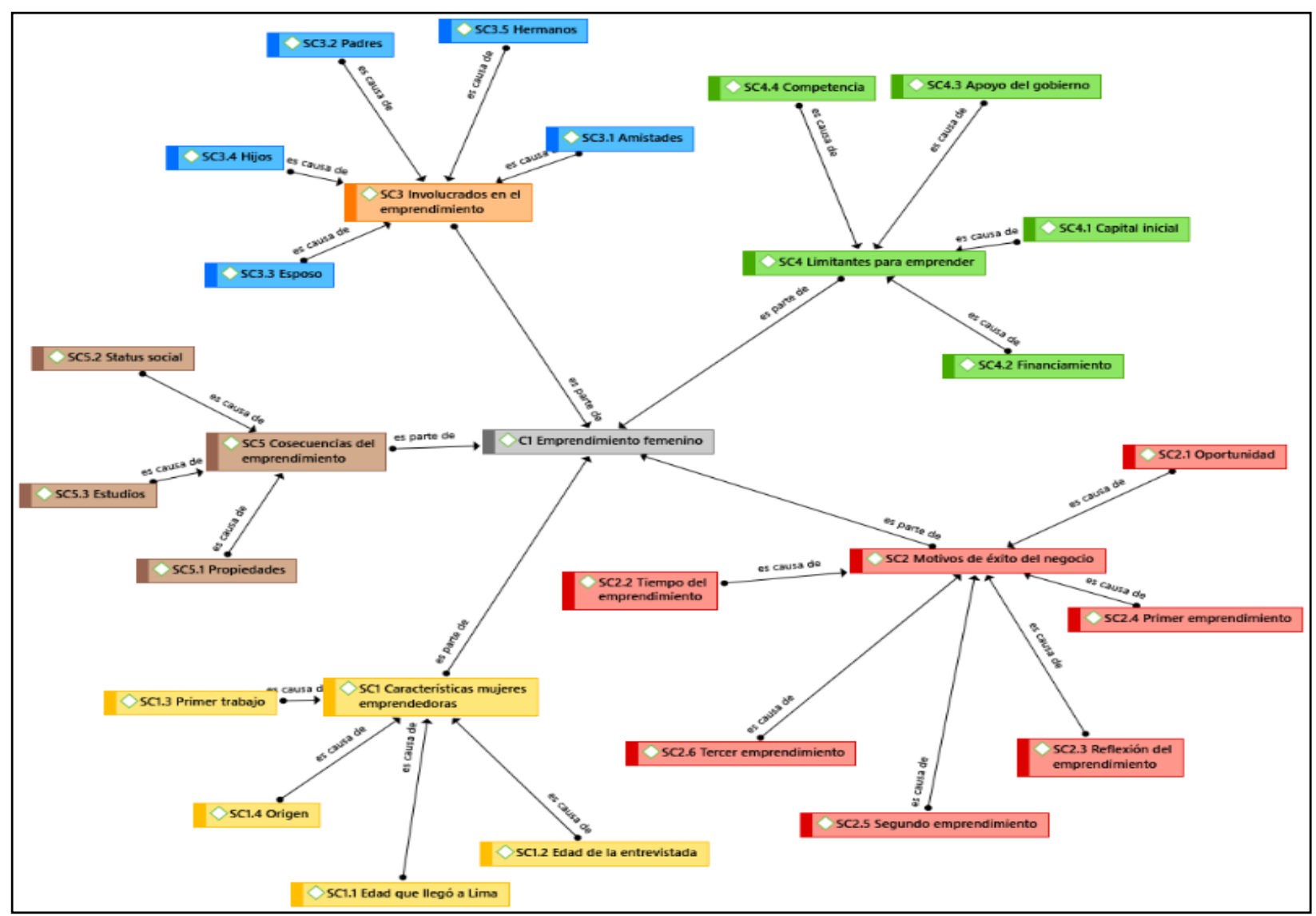




\section{Resultados y Discusión}

\section{Características de las mujeres emprendedoras}

La mayoría de las mujeres emprendedoras no son oriundas de Lima, el intervalo de edades cuando llegaron se ubicó entre 11 y 16 años. A la fecha de la recopilación de los datos el intervalo de edades se ubicó entre 26 y 49 años. El promedio de tiempo que radican en la capital es de 30 años. La mayoría realizó el trabajo de asistente del hogar a su llegada. Los lugares de origen correspondieron a Cajamarca, Huaraz, Tingo María, Ayacucho y Apurímac, como se indica en la figura 2. Las emprendedoras sostienen en conjunto que todas deben de luchar por sus sueños, ser perseverantes, observadoras, pensar en crecer personal y profesionalmente, soñar, valorar a la familia y jamás olvidarse de su origen. Para emprender no se requieren de abundante capital, ni recursos, sino que se empieza con todo aquello que se encuentra a su alcance.

\section{Figura 2}

\section{Características de las unidades informantes}

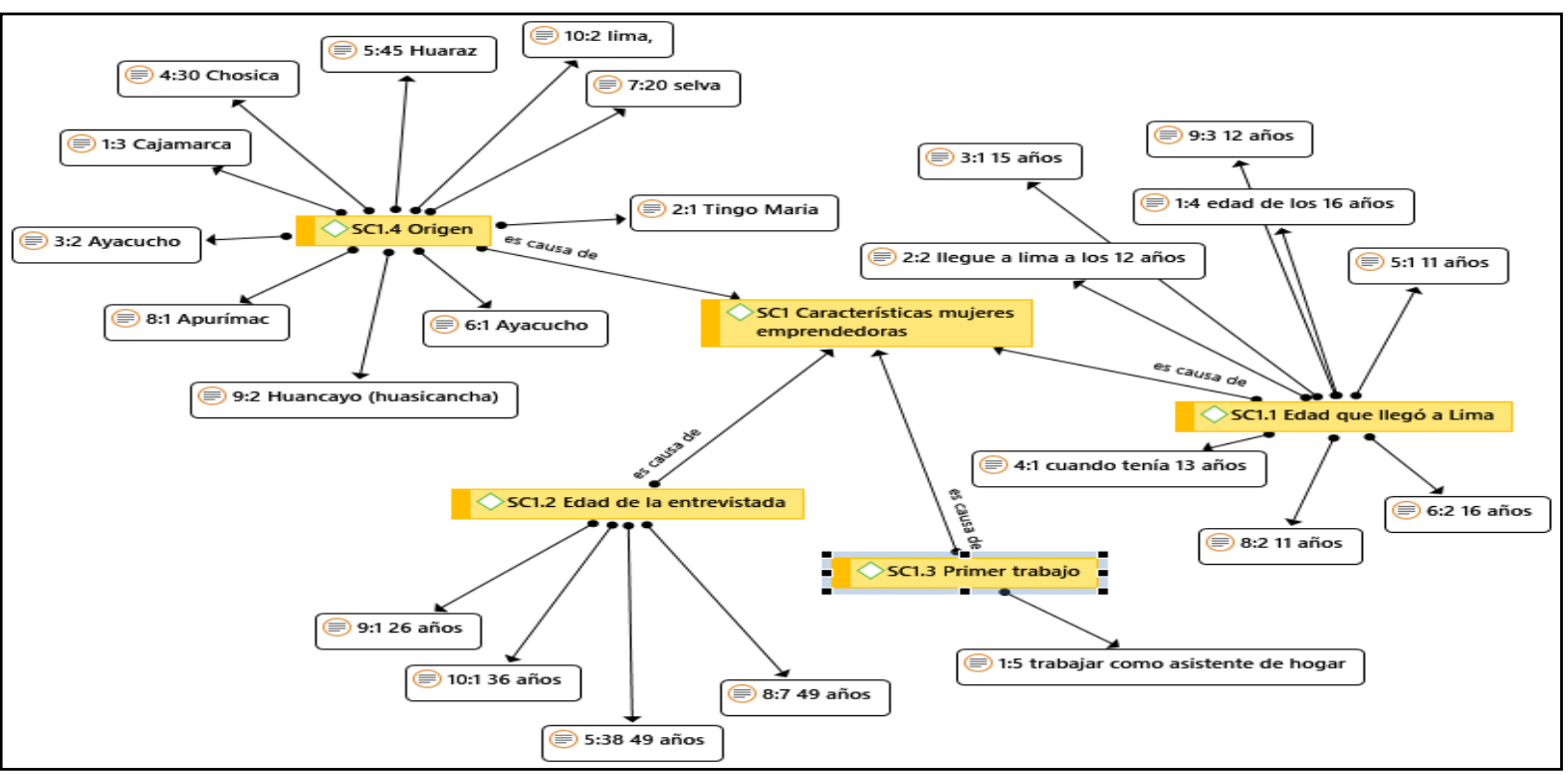

Las características de las mujeres emprendedoras según las entrevistas indican que: nunca se rinde ante diferentes circunstancias, es decir se practica la perseverancia. Por otra parte, indican que son positivas, ante una situación aparentemente mala o desfavorable, rescatan la oportunidad de mejorar el negocio. Son guerreras, persistentes, perseverantes, y siempre se levantan de los fracasos que pudieran tener, "el éxito se obtiene a través de mucho esfuerzo y de muchas caídas", desde luego al primer intento no necesariamente se logra el éxito, pero si se aprende, y las capacitaciones han aportado bastante al negocio. Al mismo tiempo consideran que los emprendimientos requieren dedicación y esfuerzo para que crezca en el tiempo, y se logre beneficios. Los valores que practican son: la honestidad, responsabilidad, puntualidad, disciplina, constancia y humanidad. 


\section{Figura 3}

\section{Características de las mujeres emprendedoras}

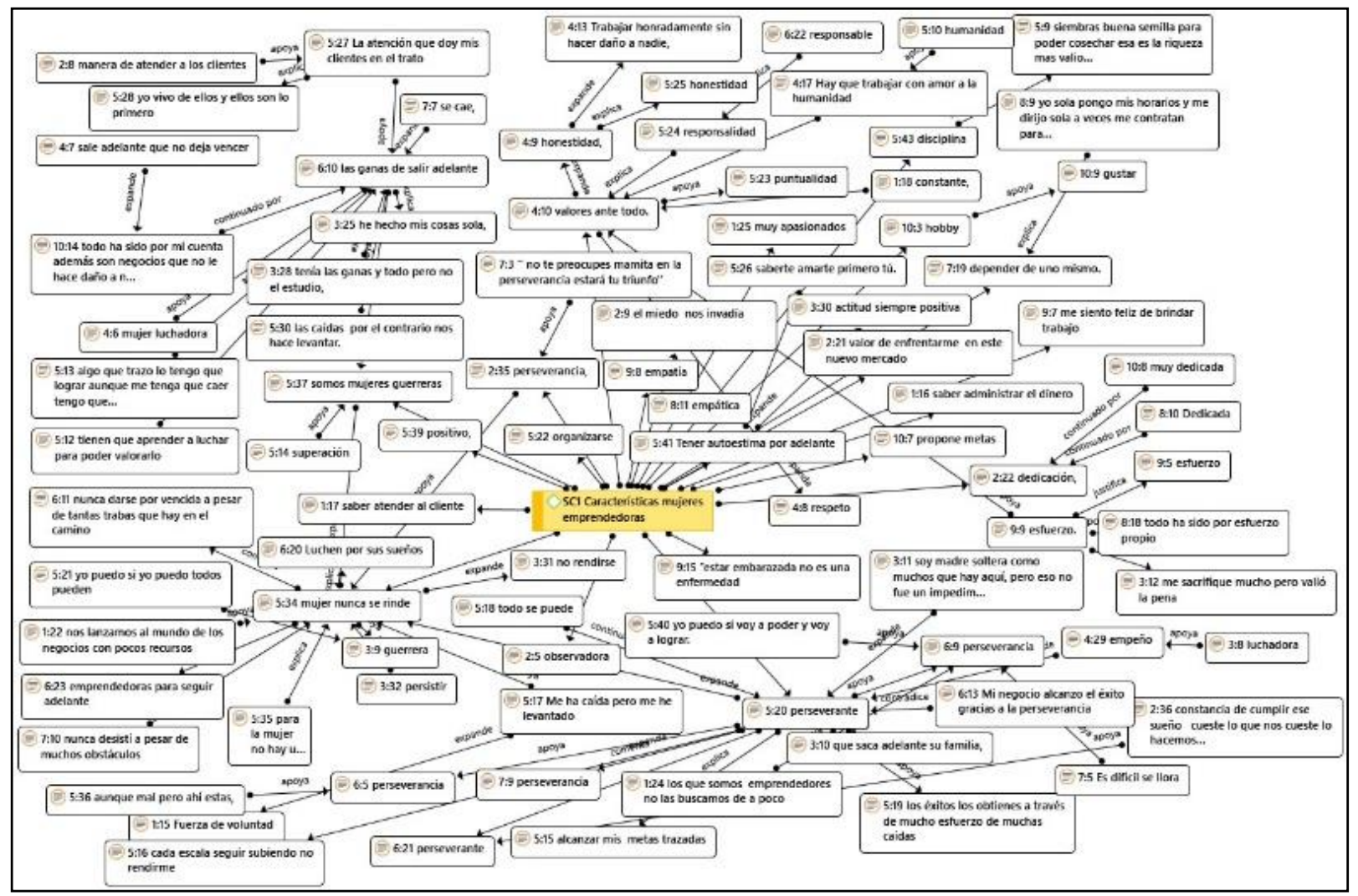

\section{Motivos de éxito}

Las mujeres afirmaron que el motivo de éxito del negocio es por la dedicación y empeño que practican día a día en sus negocios. Sostuvieron que se debe procurar tener todos los productos que requieren los clientes. La entrevistada 4 afirmó que "vendo ropa deportiva y casacas de calidad", por tal motivo es que los clientes siempre regresan; sin embargo, cuando no tienen el suficiente efectivo, optan por el abono semanal y así logre acumular la cantidad necesaria para adquirir un producto. Otros factores importantes corresponden a la limpieza del local, la sazón única, la atención, los sorteos y sobre todo debe gustarle el negocio.

Asimismo, el emprendimiento fue por oportunidad en su mayoría, la entrevistada 1 afirmó que "vende las cosas o productos que requieren los clientes", es decir satisface sus necesidades. Por otra parte, una estrategia es "aprovechar la necesidad de las personas, porque esa necesidad puede ser para ti el éxito", en este sentido las emprendedoras están atentas a los requerimientos de los clientes, por ende, la observación juega un papel importante. Una estrategia interesante que aplican es el sorteo, es decir por la compra regalan un ticket, luego en fechas determinadas se sortea productos, artefactos, entre otros, los mismos que generan expectativa en el premio, y por ello incentivan la compra. 
Asimismo, es preciso resaltar que los emprendimientos actuales no fueron los primeros, la mayoría se dedicó a la comercialización ambulatoria, sin embargo, a pesar de su inexperiencia indican que siempre buscaban "una zona estratégica" para allí vender sus productos, generar recursos económicos, ahorrar e invertir en bienes.

Las experiencias previas permitieron conocer mejor las necesidades de las personas y diseñar estrategias de posicionamiento y ventas, para lograr la rentabilidad de estas. Las mujeres entonces para ser exitosas deben de luchar, esmerarse y dar todo de sí para que su negocio o empresa se posicione, crezca y genere recursos económicos a largo plazo. El principal motivo es que su esfuerzo y crecimiento permitirá solventar a su familia, pero sobre todo generar la estabilidad emocional, así como económica (Ver figura 4).

\section{Figura 4}

\section{Motivos de éxito del negocio}

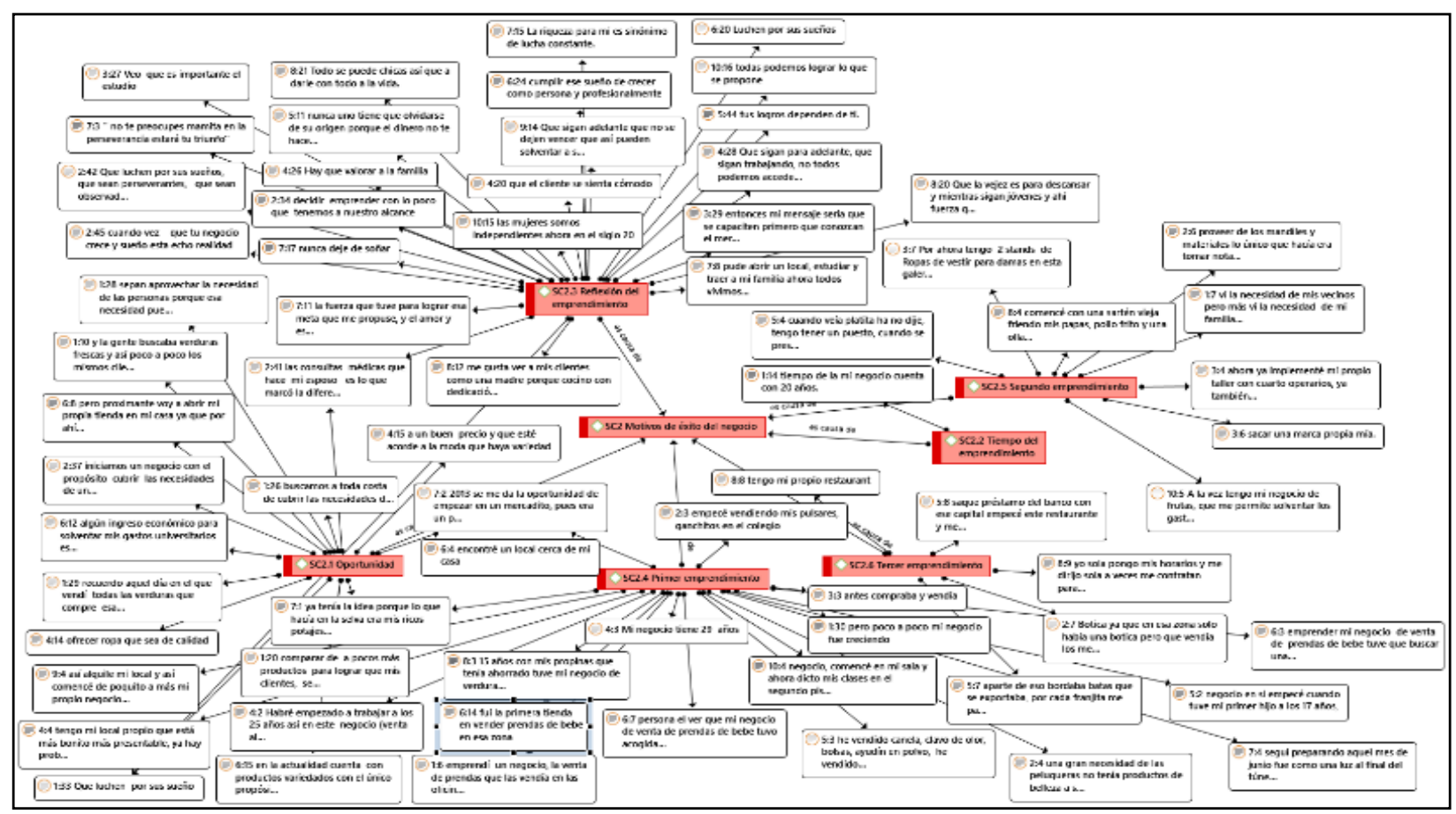

\section{Limitantes para emprender}

El emprendimiento femenino en el proceso ha tenido algunas limitaciones como: a) Factor económico, es complicado acceder a los bancos, por ello recurren a préstamos personales o familiares, se rescata el hecho que iniciaron con montos inferiores a los US 100. Un banco que apoya es Mi Banco, a pesar que las tasas de interés son elevadas, sin embargo persisten en seguir solicitando los préstamos por la prontitud del desembolso; b) Factor municipal, la licencia tarda demasiado para su obtención, los trámites son burocráticos y exigen cada vez más documentos que no fueron requeridos al inicio del proceso; c) Gobierno central - Ministerios, sostuvieron que 
nunca las apoyaron, sin embargo una entrevista indicó que le comentaron que las municipalidades brindan talleres, capacitaciones, pero no ha logrado tener la información respectiva. Otra entrevistada indicó que el Ministerio de trabajo la capacitó en atención al cliente y manejo de las mercaderías o inventarios; d) Competencia, existen negocios posicionados y con una mejor infraestructura, que les permite ser más competitivas, pero a pesar de ello emprendieron sus negocios, consideran que la clave es la atención, como se aprecia en la figura 5.

\section{Figura 5}

\section{Limitantes para emprender}

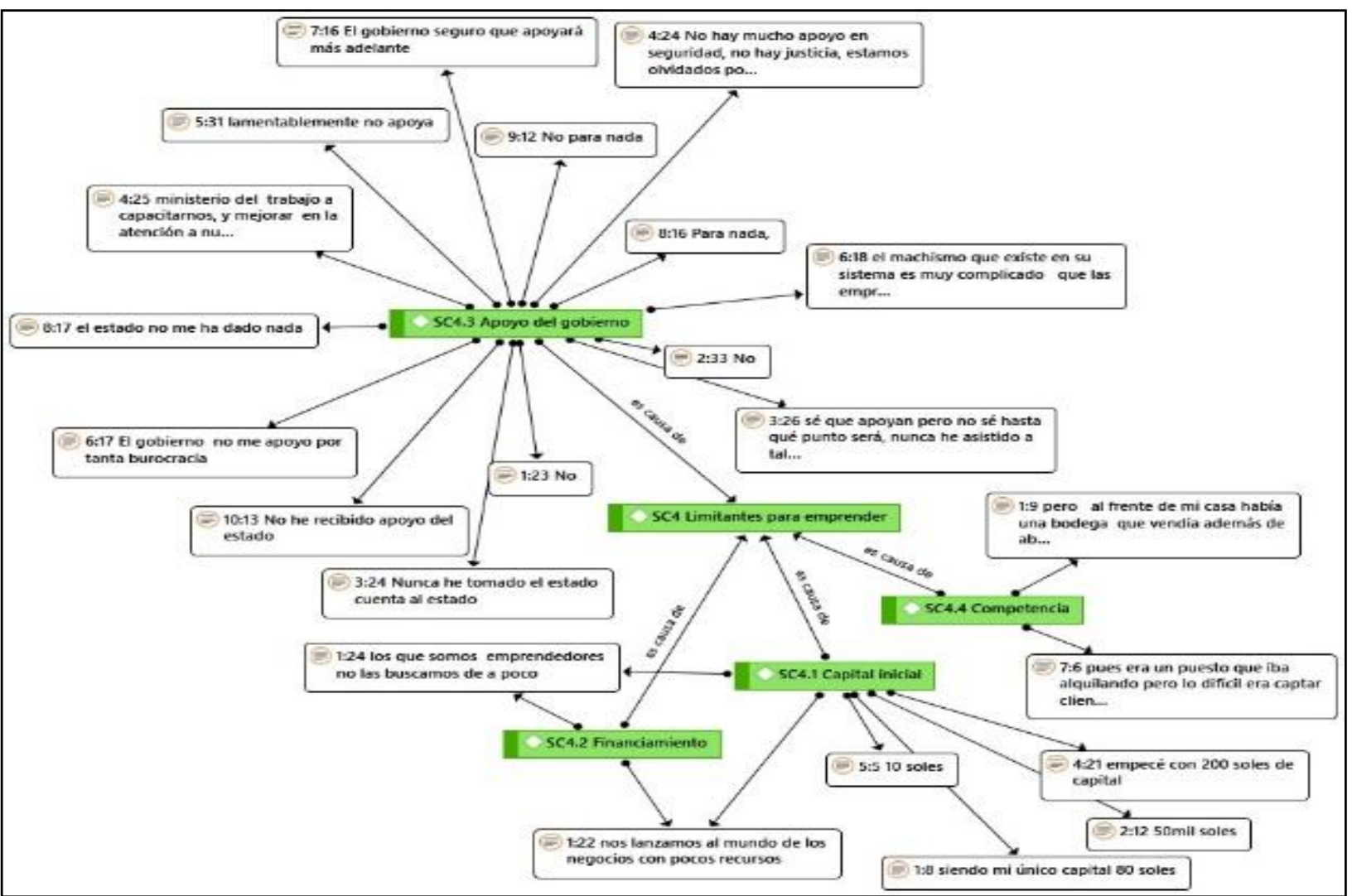

\section{Involucrados y consecuencias}

En el emprendimiento femenino están involucrados en su mayoría su esposo e hijos. El conyugue apoya en el establecimiento con labores de atención al público, alguno de ellos optó por renunciar a su empleo para que se involucre al $100 \%$ en el negocio Los hijos también apoyan en la supervisión y asesoramiento, porque muchos están estudiando a nivel universitario, y con una perspectiva diferente aportan al crecimiento del negocio, como se aprecia en la figura 6.

El emprendimiento ha permitido a las mujeres lograr la independencia económica, viajar, capacitarse constantemente, generar empleo para terceros, crecimiento del negocio, satisfacer sus propias necesidades como mujer, educar a sus hijos en colegios particulares, casa y auto propio, 
talleres equipados, apoyar a su familia. En resumen, se aprecia una economía sostenible, además de la generación de la riqueza personal y familiar, como se aprecia en la figura 6.

\section{Figura 6}

\section{Involucrados en el emprendimiento}

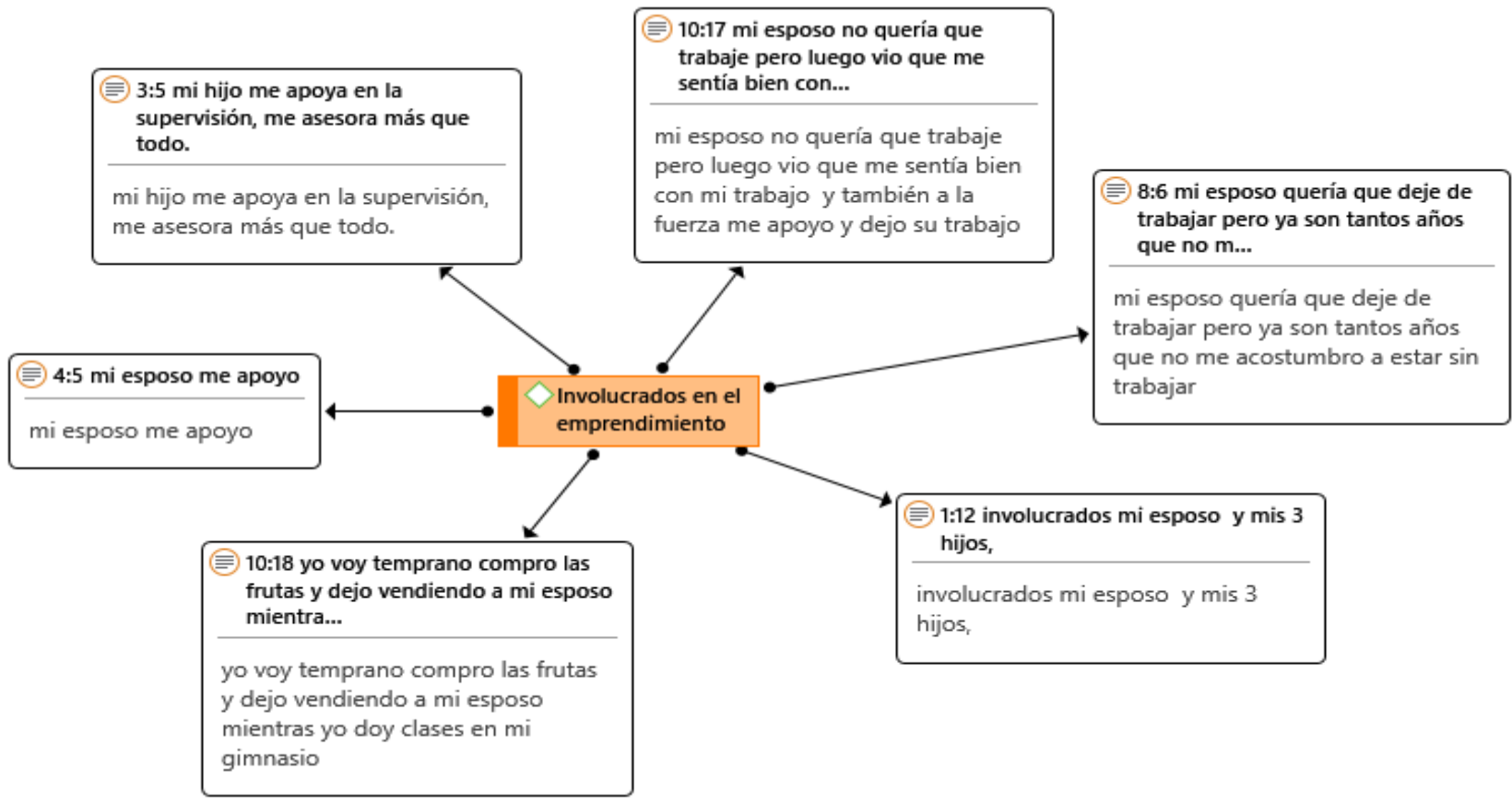

En el estudio, observamos que las mujeres emprenden, en su mayor parte, estos resultados armonizan con el Global Entrepreneurship Monitor Perú 2017-2018 (GEMP) porque el 62,3\% de los peruanos están motivados por la oportunidad y con la Pontificia Universidad Católica de Chile (2018). A pesar del empirismo, las mujeres observan continuamente las necesidades de sus clientes y de la competencia, para implementar nuevos productos o servicios, sin embargo, si el negocio no es rentable, entonces pasan a otro turno.

El espíritu empresarial para las mujeres es muy arriesgado; los bancos no brindan acceso a financiamiento. Los resultados concuerdan con Ascher (2012), Kumar (2015) y Chowdhury (2018) debido a que las mujeres no acceden fácilmente al financiamiento otorgado por bancos $\mathrm{y}$ financieras, sin embargo, cuando logran calificar, estos se otorgan con mesura.

Del mismo modo, las mujeres tienen miedo de fracasar; esta última situación armoniza con el Instituto de Empresa (2019) y el GEMP (2018) porque el 30,7\% de los peruanos cree que el miedo al fracaso restringe el emprendimiento. Al mismo tiempo, el nivel educativo de las mujeres emprendedoras era principalmente educación superior técnica; esta situación es consistente con Perú (GEMP, 2018) porque el 26\% de los emprendedores tienen el mismo nivel de educación.

Por otro lado, el hecho de emprender permitió a las mujeres mejorar su condición social y prestigio; este resultado es consistente con los ciudadanos de Tanzania (Kabote, 2018), Asia y 
Oceanía (GEMP, 2018). La familia apoya el emprendimiento y participa en la toma de decisiones, también aumentó los recursos económicos, y por eso adquirió bienes y vehículos. Asimismo, las mujeres se comprometieron a lograr la independencia económica; esta situación se coordina con Eib y Sieget (2019). La característica principal es la perseverancia y la motivación para satisfacer no solo las necesidades, sino también la familia. Finalmente, el estudio aporta a las ciencias sociales con características, limitaciones y consecuencias del emprendimiento femenino en Villa El Salvador.

\section{Conclusiones}

Las mujeres emprendedoras se caracterizan por ser perseverantes, positivas y nunca desmayarse ante cualquier situación adversa. Sin embargo, el hecho del emprendimiento no las limita en las actividades del género. El éxito del emprendimiento se debe básicamente a la dedicación y perseverancia que le dedican al negocio, además de identificar la oportunidad. Una limitación trascendental es el acceso a préstamos para inversión inicial, así como permisos municipales, que resultan ser burocráticos. Una vez más se ratifica que el apoyo familiar es fundamental para alcanzar el éxito personal y empresarial. Un negocio bien administrado se evidencia en la rentabilidad y el aumento de la riqueza personal.

Las emprendedoras aprendieron de finanzas, porque ante la necesidad del cliente por el producto, y la no disponibilidad de los recursos, diseñaron la política de pago semanal. De tal forma que, cuando el cliente acumule el monto del producto, entonces hace entrega del mismo, y con ello genera la lealtad, y así apuesta por ventas futuras.

Por otra parte, las emprendedoras coinciden en el hecho que el emprendimiento actual no es el primero, tuvieron antes otras actividades comerciales, las cuales no les generaba el beneficio esperado, o en otros casos solo era por temporadas. Sin embargo, reconocen que la experiencia previa las ayudó bastante para el emprendimiento actual. También se hace necesario que el gobierno de turno en conjunto con la banca privada y las ONGs diseñe productos especiales para financiar los emprendimientos en general, considerando un costo de capital menor que un préstamo personal o de consumo, porque no permite el crecimiento sostenible del negocio.

Finalmente, los estudios futuros deben seguir abordando el tema del emprendimiento femenino desde una perspectiva del enfoque cuantitativo y diseñar modelos para explicar la motivación, características y consecuencias de tal decisión, además del beneficio para la familia.

\section{Referencias bibliográficas}

Amin, A. (2018). Women Entrepreneurship and SMEs of Online Clothing Business in Dhaka City. $\begin{array}{llll}\text { Universal Journal of } & \text { Management, } & 6 & \text { (10), 359-372. }\end{array}$ https://doi.org.10.13189/ujm.2018.061001

Ascher, J. (2012). Female Entrepreneurship - An Appropriate Response to Gender Discrimination. Journal of Entrepreneurship, Management and Innovation, 8 (4), 97-114. https://bit.ly/33o00nM 
Barco, B. y Carrasco, A. (2018). Explicaciones causales en la investigación cualitativa: elección escolar en Chile. Revista Internacional de Investigación en Educación, 11 (22), 113-124. https://doi.org/10.11144/Javeriana.m11-22.ecic

Bouzekraoui, H. y Ferhane, D. (2017). An Exploratory Study of Women's Entrepreneurship in Morocco. Journal of Entrepreneurship: Research \& Practice, 1-19. https://doi.org/10.5171/2017.869458

Casson, M. (2001). The entrepreneur: an economic theory. Brookfield-USA: Gregg Revivals.

Castillo, A. (1999). Estado del arte en la enseñanza del emprendimiento. Programa Emprendedores como creadores de riqueza y desarrollo regional Intec. Chile: Intec. https://bit.ly/3ITxLxt

Chowdhury, S. (2018). Financing female entrepreneurs in cottage, micro, small, and medium enterprises: Evidence from the financial sector in Bangladesh 2010-2018. Asia \& the Pacific Policy studies. Wiley, 6, 397-416. https://doi.org/10.1002/app5.286

Da silva, S., Esper, A. Bencciveni y Chagas, D. (2015). Empreendedorismo feminino: um estudo fenomenológico. Navu, 5 (2), 6-13. https://bit.ly/3J3ZpZ0

Dornela, J. (2001). Empreendedorismo. Brasil: Editora Campus Ltda - Una empresa Elsevier.

Drucker, P. (1985). La innovación y el empresario innovador. Barcelona: Edhasa.

Eib, C. y Sieget, S. (2019). Is Female Entrepreneurship Only Empowering for Single Women? Evidence from France and Germany. Social sciences, 8, 2-9. https://bit.ly/3J24ZuY

Guerrero, L., Gómez, E. y Armenteros, M. (2014). Mujeres emprendedoras: Similitudes y diferencias entre las ciudades de Torreón y Saltillo, Coahuila. Revista Internacional Administración \& Finanzas, 7(5), 77-90. https://bit.ly/3e3633g

Global Entrepreneurship Monitor. (2017). Women's Entrepreneurship 2016/2017 Report. London: GEM.

Global Entrepreneurship Monitor: Perú 2017-2018 (2018). Lima: Universidad ESAN

Gutiérrez, M. (2006). Teoría de los rasgos. Contribución a la interpretación del comportamiento del empresario. Gestión, 5 (2), 30-35.

Hernández, R., Fernández, C. y Baptista, M. (2014). Metodología de la investigación. México: McGraw-Hill.

Huilcapi, M. Castro G. y Jácome, G. (2017). Motivación: las teorías y su relación en el ámbito empresarial. Dominio de las Ciencias, 3 (2), 311-333. https://bit.ly/3J27wFm

Instituto de Empresa. (2019). Informe GEM España 2018 - 2019. Santander: Editorial de la Universidad de Cantabria, 2019.

Jia, J., Parvin, L. y Rahman, M. (2012). Determinantes of women micro entrepreneurship: An empirical investigation in rural Bangladesh. International Journal of Economics and Finance, 4 (5), 254-260. https://bit.ly/3pbdlIN

Kabote, S. (2018). Influence of women entrepreneurs' characteristics on personal well-being in Arumeru District in Tanzania. African Journal of Business Management, 12, (11), 316328. https://doi.org/10.5897/AJBM2018.8558

Kargwell, S. (2012). A comparative study on gender and entrepreneurship development: still a male's world within UAE cultural context. International Journal of Business and Social Science, 3, (6), 44-55.

Kumar, P. (2015). A study on women entrepreneurs in India. International I Journal of Applied Science \& Technology, 5 (5), 43-46. https://bit.ly/3e5J6wr

McKenzie, B., Ugbah S., Smothers, N. (2007). Who is an entrepreneur? Is it still the wrong question? Academy of Entrepreneurship Journal, 13, 23-43. https://bit.ly/323atF9 
Mehrad, A. y Tahriri, M. (2019). Comparison between Qualitative and Quantitative Research Approaches: Social Sciences. International Journal for Research in Educational Studies, 5, (7), 1-7. https://gnpublication.org/index.php/es/article/view/998

Navarro, E. Jiménez, E., Rappoport, S. y Thailiez, B. (2017). Fundamentos de la investigación y la innovación educativa. España: Universidad Internacional de La Rioja S.A., 2017.

Nueno, P. (1999). Emprendiendo: el arte de crear empresas y sus artistas, Bilbao: Deusto.

Numa, N. y Márquez, R. (2019). Los semilleros como espacios de investigación para el investigador novel. Propósitos y Representaciones, $7 \quad$ (1), 230-248. http://doi.org/10.20511/pyr2019.v7n1.289

Okuda, M. y Gómez-Restrepo, C. (2005). Metodología de investigación y lectura crítica de estudios. Revista Colombiana de Psiquiatría, XXXIV, (1), 118-124. https://www.redalyc.org/pdf/806/80628403009.pdf

Peris, M., Peris, F. y Ribeiro, D. (2010). Capacidades y mujer emprendedora. Mujeres y Economía, (852), 153-165. https://bit.ly/2ShWotQ

Pontificia Universidad Católica de Chile. (2018). Programas de apoyo al emprendimiento femenino en la Alianza del Pacífico, Santiago de Chile. Universidad Católica de Chile.

Prince M. y Felder, R. (2006). Inductive teaching and learning methods: definitions, comparisons, and research bases. J. Engr. Education, 95 (2), 123-138. https://bit.ly/3FaiSVB

Rodríguez, A. y Pérez, A. (2017). Métodos científicos de indagación y de construcción del conocimiento. Revista de la Escuela de Administración de Negocios, 82, 179-200. https://doi.org/10.21158/01208160.n82.2017.1647

Rosa, S., Souza, V. y Loch, A. (2011). Comportamentos empreendedores: por que as mulheres empreendem?, Santa Catarina: Pandion.

Sánchez, A. (2006). Peter Druker, innovador maestro de la administración de empresas. Cuadernos Latinoamericanos de Administración, 2 (2), 69-89. https://bit.ly/3J5nGxB

Serida, J., Guerrero, C., Alzamora, J., Borda, A. y Morales, O. (2018). Global Entrepreneurship Monitor: Perú 2017 - 2018. Lima: ESAN

Vásquez, J. (2016). El emprendimiento empresarial: La importancia de ser emprendedor. España: IT Campus Academy.

Villagómez, F. (1994). Douglas C. North su contribución hacia una teoría del cambio institucional. Política y Gobierno, 1, (2), 391-400. https://bit.ly/3qd7fHb

Villalobos, G. y Pedroza, R. (2009). Perspectiva de la teoría del capital humano acerca de la relación entre educación y desarrollo económico. Tiempo de Educar, 10 (20), 273-306. https://bit.ly/3seWFC0 